\section{Laudation to W. Neupert}

The Jury has decided to award this year's Heinrich Wieland Prize to Prof. Walter Neupert in recognition of his successful research work on mitochondria.

As well as the nucleus, body cells contain organelles which perform various functions. The most numerous of these are the mitochondria. They are enclosed in a double membrane system which contains the enzymes for essential processes in the cell, e.g. oxidative phosphorylation, the citrate cycle and the degeneration of fatty acids.

The mitochondria reproduce by growth and division, which means inter alia the incorporation of newly synthesized proteins into the growing organelles. The biosynthesis of mitochondrial proteins in governed by two genomes, that of the mitochondrion itself, which accounts for only a small number of proteins however, and - mainly - by the genome of the cell nucleus. So the question arises as to how the many hundreds of nucleus-coded proteins pass in specific ways through the lipid bar riers of the membranes and subsequently join to form functional structures between the membranes and in the interior, the matrix.
Professor Walter Neupert has studied and determined the molecular mechanisms of this passage through the lipid layers in the several examples. Amongst other things, he discovered that adenosine triphosphate and heat-shock proteins (hsp) perform several functions as "molecular motors". This includes unfolding of the polypeptide chains into a conformation capable of passing through the membrane, and the passage through the membrane to meet with other components which facilitate refolding. The newly formed proteins must be transported via complex sorting and transport routes to their proper places, some of which have also been identified.

Professor Neupert was born in Munich in 1939, and obtained doctorates in chemistry in 1968 and in medicine in 1970 at Munich University where, from 1969-1972 he worked as a Research Assistant at the Institute of Physiological Chemistry and from 1972-1977 as a lecturer. From 1977-1983 he was Professor at the Institute of Biochemistry at Göttingen University. Since 1983 he has held a chair at the Institute of Physiological Chemistry, Physical Biochemistry and Cell Biology at the University of Munich.

\title{
Transport of proteins across mitochondrial membranes
}

\author{
W. Neupert \\ Institut für Physiologische Chemie, Physikalische Biochemie und Zellbiologie, Universität München, D-80336 München, Germany
}

\begin{abstract}
The vast majority of proteins comprising the mitochondrion are encoded by nuclear genes, synthesized on ribosomes in the cytosol, and translocated into the various mitochondrial subcompartments. During this process proteins must cross the lipid membranes of the mitochondrion without interfering with the integrity or functions

Abbreviations: $\mathrm{OM}=$ outer mitochondrial membrane; $\mathrm{IM}=$ inner mitochondrial membrane; IMS = mitochondrial intermembrane space; $\mathrm{OMV}=$ outer membrane vesicles; $\mathrm{AAC}=$ $\mathrm{ADP} / \mathrm{ATP}$ carrier $; \mathrm{PiC}=$ phosphate carrier; $\mathrm{DHFR}=$ mouse cytosolic dihydrofolate reductase; $F_{1} \beta=$ subunit $\beta$ of $F_{1} F_{0^{-}}$ ATPase; $\mathrm{MPP}=$ mitochondrial processing peptidase
\end{abstract}

of the organelle. In recent years an approach combining biochemical, molecular, genetic, and morphological methodology has provided insights into various aspects of this complex process of intracellular protein sorting. In particular, a greater understanding of the molecular specificity and mechanism of targeting of mitochondrial preproteins has been reached, as a protein complex of the outer membrane which facilitates recognition and initial membrane insertion has been identified and characterized. Furthermore, pathways and components involved in the translocation of preproteins across the two mitochondrial membranes 
are being dissected and defined. The energetics of translocation and the processes of unfolding and folding of proteins during transmembrane transfer are closely linked to the function of a host of proteins known as heat-shock proteins or molecular chaperones, present both outside and inside the mitochondrion. In addition, the analysis of the process of folding of polypeptides in the mitochondrial matrix has allowed novel and unexpected insights into general pathways of protein folding assisted by folding factors. Pathways of sorting of proteins to the four different mitochondrial subcompartments - the outer membrane $(\mathrm{OM})$, intermembrane space, inner membrane (IM) and matrix - are only partly understood and reveal an amazing complexity and variation. Many additional protein factors are involved in these latter processes, a few of which have been analyzed, such as cytochrome $c$ heme lyase and cytochrome $c_{1}$ heme lyase, enzymes that catalyze the covalent addition of the heme group to cytochrome $c$ and $c_{1}$ preproteins, and the mitochondrial processing peptidase which cleaves signal sequences after import of preproteins into the matrix. Thus, the study of transport of polypeptides through the mitochondrial membranes does not only contribute to the understanding of how biological membranes facilitate the penetration of macromolecules but also provides novel insights into the structure and function of this organelle.

Key words: Protein transport - Mitochondria Molecular chaperones - Protein folding - Preprotein receptors

Protein translocation across and into biological membranes is a key problem in cell biology. It represents the initial step in protein secretion, a process common to all prokaryotic and eukaryotic cells. Protein translocation is also a central issue in the biogenesis of cell organelles, in particular of mitochondria and chloroplasts. It is generally accepted that these two organelles originate from prokaryotic endosymbionts since they impressively display traits of stunning similarity to their presumed prokaryotic ancestors, the purple bacteria and the blue green algae. There is also little reason to doubt that, during the transition from the endosymbiotic stage to the present organellar state, mitochondria and chloroplasts transferred most of their genetic material to the host's genome, i.e., to the cell nucleus. As a consequence the proteins once made in the endosymbiont must now be transported into the organelle. Many hundreds of different proteins, which represent a substantial portion of total cellular protein mass, must undergo this process to reach their functional location.

Thus, eukaryotic proteins are synthesized almost exclusively on ribosomes in the cytosol; consequently, however, many proteins must be delivered to various intracellular compartments and to the exterior of the cell. This implies that translocation of proteins across the lipid phase of intracellular membranes must take place. In the case of both secretory and resident proteins of the endomembrane system the obstacle to overcome is the membrane of the endoplasmic reticulum. In the case of mitochondria and chloroplasts translocation across the boundary membranes must be emcompassed by proteins destined to those organelles. This complex situation raises a number of intriguing questions. How are newly synthesized proteins recognized by specifically the organelle into which it must be imported? How can a polypeptide chain cross biological membranes which are designed to be impermeable to proteins, and how is the integrity of the membranes, in terms of enzymatic function and selective ion permeability, maintained during this process? What are the driving forces for the translocation process? What are the folding states of a protein when it is recognized by an organelle, when it is passing through one or two membranes, and how do polypeptide chains become folded after completion of transport? How are newly imported polypeptides sorted to the various subcompartments of an organelle, and how are they assembled to supramolecular functional structures?

The investigation of the processes involved in the transport of polypeptides across the membranes of mitochondria has provided fragmentary but promising answers to these questions. A number of mitochondrial components have been identified, and pathways have been uncovered which allow initial glimpses into the molecular details of what appears to be one of the most complex reactions in cell biology presently under study.

\section{Recognition of precursor proteins by mitochondria}

Mitochondria can be divided into two membraneous and two aqueous subcompartments: (a) the $\mathrm{OM}$ and the IM with its invaginations, the cristae membranes, and (b) the matrix space bounded by the IM and the intermembrane space (IMS) between the OM and the IM. Newly synthesized mitochondrial protein is able to "perceive" only the surface of the OM although most of them must reach an internal subcompartment. Clearly, there 
must be devices to target polypeptides selectively to the mitochondria. Newly synthesized mitochondrial proteins carry specific "addressing signals" or "targeting signals." In most cases these signals are present as amino-terminal peptide extensions, also called "prepeptides," which are cleaved off by the mitochondrial processing peptidase in the matrix after import. They comprise between ca. 15 and 70 amino acid residues [2, 24]. Comparison of the presequences of different mitochondrial proteins reveals practically no sequence similarity. There are, however, common themes in these prepeptides, namely they are hydrophilic, and they display a high frequency of positively charged residues and an almost complete absence of negatively charged residues. Furthermore, mitochondrial prepeptides have a tendency to form $\alpha$-helical amphipathic structures [26, 53]. This points to the possibility that the signal represents a structural motif rather than a sequence motif. Those mitochondrial proteins which are synthesized without a presequence must contain import information in their interior; however, very little is known about these signals $[41,49,62]$.. So far all proteins of the OM and some proteins of the IMS and the IM lack cleavable signals.

How are these signals decoded by the mitochondrion? Import into mitochondria can be reconstituted by using preproteins synthesized in cell-free systems programmed with specific cDNAs and incubating them with isolated mitochondria (Fig. 1). Under appropriate conditions including the presence of cofactors such as ATP and membrane potential generating substrates, precursors become translocated into the mitochondria and, in the case of presequence-containing preproteins, proteolytically processed. It was noticed early on that pretreatment of intact isolated mitochondria with proteases strongly reduces their ability to import preproteins. This suggested the existence of surface components which mediate recognition of preproteins [72, 73]. Hence, this observation opened the possibility of identifying such components. Antibodies were generated against a number of OM proteins and were analyzed for their ability to inhibit import. Two components were identified and eventually cloned and sequenced (Fig. 2). Mitochondrial outer membrane protein of $19 \mathrm{kDa}$ molecular mass (MOM19) is required for the import of practically all preproteins analyzed $[57,63]$. Therefore, it is believed to constitute the main import receptor of mitochondria. Neurospora crassa cells in which MOM19 is depleted by inactivation of the gene show a virtually complete block of growth, and mitochondria isolated from these cells

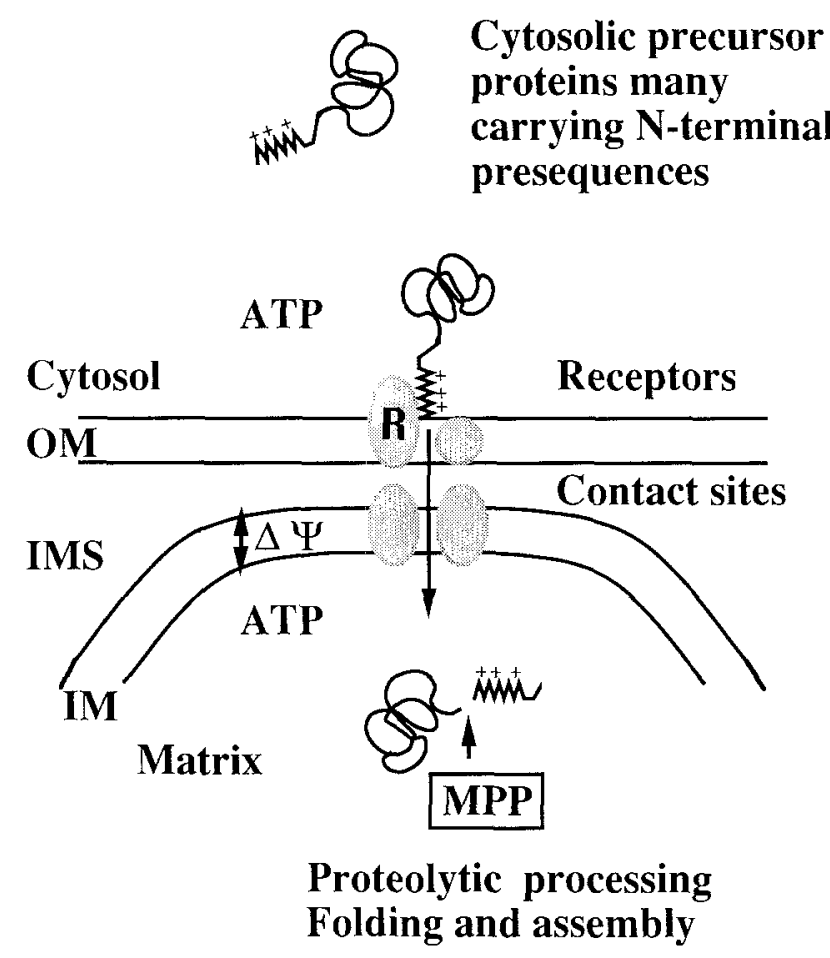

Fig. 1. Simplified scheme of protein translocation through mitochondrial membranes. $\Delta \Psi$, Electrical membrane potential; $O M$, outer membrane; IMS, intermembrane space; $I M$, inner membrane; $M P P$, mitochondrial processing peptidase

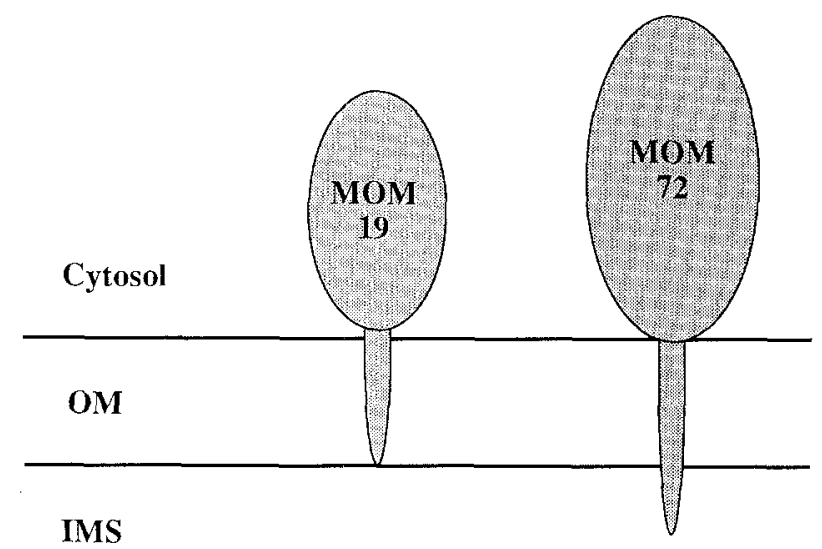

Precursors all tested AAC, PiC recognized: (except cytochrome c) (cytochrome $c_{1}$ )

Phenotype respiration minor reduction of $\begin{array}{lc}\text { of deletion } & \text { deficient, very slow } \\ \text { mutant: } & \text { growth }\end{array}$

Number of TPR motifs:

1

6-7

Fig. 2. Mitochondrial import receptors. $O M$, Outer membrane; $I M S$, intermembrane space; TPR motif, tetratricopeptide motif; $A A C, \mathrm{ADP} / \mathrm{ATP}$ carrier; $P i C$, phosphate carrier 
are incompetent for import of most but not all preproteins, in particular of those containing N-terminal extensions [22]. Mitochondria in the mutant cells have lost the cristae membranes and their membrane bound cytochromes. MOM19 is thus an important although not essential component of the import machinery. The second component identified was MOM72, antibodies against which inhibited the import of a subset of preproteins, including the very abundant IM proteins ADP/ATP carrier (AAC) and phosphate carrier (PiC) $[12,29,64]$. In the absence of MOM72 these proteins can be imported via MOM19. Deletion of MOM72 in yeast had very little effect on the growth of cells or the formation of mitochondria [66]. Thus, the mitochondrial surface receptors have overlapping specificities and are important to the cell to different extents.

Both MOM19 and MOM72 are integrated into the outer mitochondrial membrane by a hydrophobic sequence close to the $\mathrm{N}$-terminus $[25,57,66]$. They expose a hydrophilic domain into the cytosol which may be involved in the binding of precursor proteins (Fig. 2). The cytosolic domain of MOM72 was expressed in Escherichia coli and purified to homogeneity. This fragment of MOM72 was able to compete for the import of a subset of preproteins, including the $\mathrm{AAC}$ and $\mathrm{PiC}$, and was able to bind directly to these preproteins (Schlossmann et al., submitted for publication). This suggests that the cytosolic domain of MOM72 (and probably MOM19) functions as receptors by binding targeting signals of preproteins in the cytosol.

\section{The translocation machinery of the outer mitochondrial membrane}

Analysis of preprotein targeting in $N$. crassa mitochondria suggested that the surface receptors are functionally linked to a general insertion pore in the OM. Coimmunoprecipitation experiments with antibodies against MOM19 and MOM72 revealed the existence of a complex in the OM with four other protein components, MOM22, MOM38, MOM7, and MOM8 [36, 44] (Fig. 3). This complex is stable in the presence of mild detergents, such as digitonin, but is dissociated by stronger detergents, such as Triton X-100. MOM22 is a protein that spans the OM once and exposes domains both into the cytosol and the IMS. Antibodies against MOM22 inhibit the import of practically all preproteins. A detailed analysis suggests that preproteins are transferred from MOM19 and MOM72 to MOM22, whence they enter the putative transport pore [37].

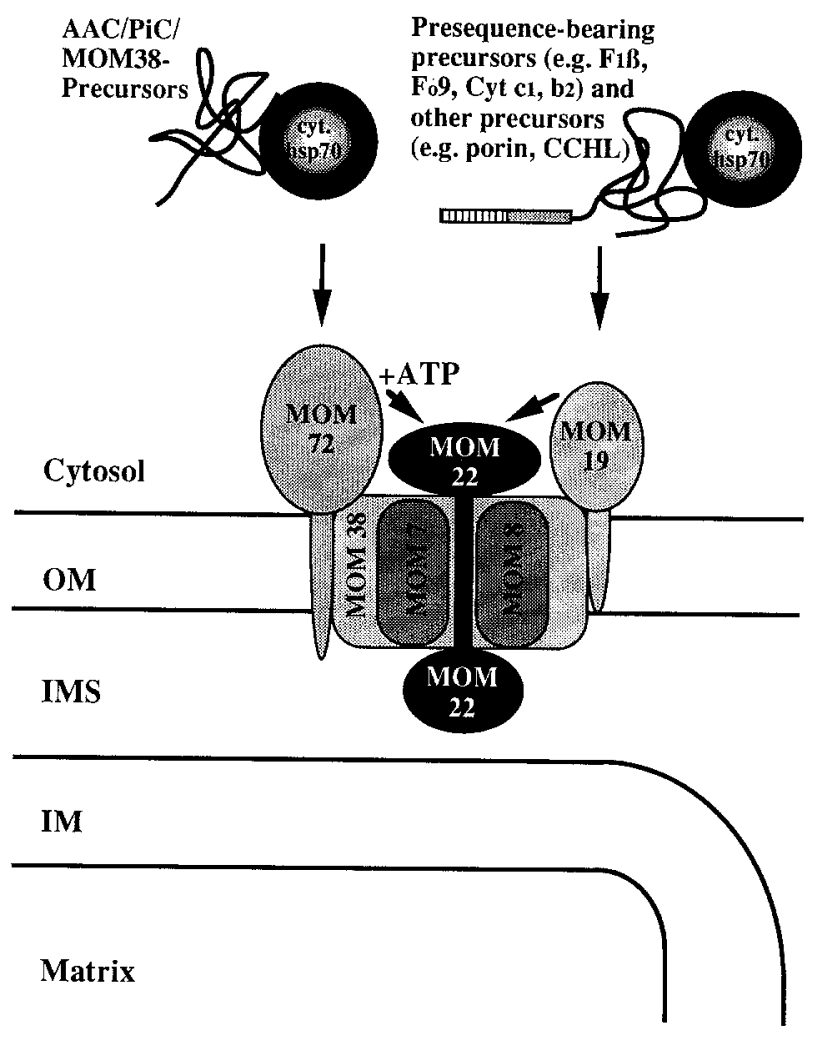

Fig. 3. Mitochondrial receptor-GIP complex (outer membrane pore complex). $F_{1} \beta$, Subunit $\beta$ of $\mathrm{F}_{1} \mathrm{~F}_{0}$-ATP synthase; $F_{0} 9$, subunit 9 of $\mathrm{F}_{1} \mathrm{~F}_{0}$-ATP synthase; $C y t c_{1}, b_{2}$, cytochromes $c_{1}$ and $b_{2}$; $C C H L$, cytochrome $c$ heme lyase; cyt.hsp 70 , cytosolic hsp 70

MOM38 in $N$. crassa (and the homolog in the yeast Saccharomyces cerevisiae, ISP42) appears to have a central function in constituting a protein translocation channel across the OM $[3,36]$ Polypeptide chains in transit across both mitochondrial membranes have been chemically crosslinked to this integral membrane protein $[65,68]$. Although MOM38 is not a very hydrophobic protein, it appears to span the $\mathrm{OM}$ at least twice and may thus represent an important constituent of a hydrophilic pore that facilitates the passage of both hydrophobic and hydrophilic preproteins. MOM7 and MOM8 are the smallest components of the complex; they are found in close proximity to translocating chains by cross-linking [65]. It is speculated that they contribute to the formation of the translocation pore. In yeast, a gene termed ISP6 was cloned that seems to interact with MOM38/ ISP42 and may be related to one of the $N$. crassa components [35].

In summary, the OM contains a complex consisting of at least six membrane-integrated components which facilitate both recognition and membrane translocation of preproteins. This suggests 
that membrane translocation of polypeptide chains needs a proteinaceous machinery. Whether this machinery forms a true channel surrounded exclusively by proteins, or whether membrane lipids function as constituents of this channel is presently an open question. The similarities of this machinery in organisms as distantly related as $N$. crassa and $S$. cerevisiae indicate that its basic outline is similar in all eukaryotes.

\section{Transport of proteins across both outer and inner membranes}

It was realized quite early that the transport of proteins into the matrix space occurs in a coordinated fashion across both OM and IM. Intermediates have been accumulated whose $\mathrm{N}$-terminus had reached the matrix space (and was processed by mitochondrial processing peptidase, MPP) whereas C-terminal parts of the preprotein were still exposed to the cytosolic compartment [56]. A number of conditions lead to arrest of translocation in such a fashion, for example, import at lower temperature, reduction of the ATP level in the extramitochondrial space, and presence of a tightly folded domain at the C-terminus of a preprotein $[7,13,50$, 70]. The common theme of these different conditions is that they disfavor unfolding of preproteins in the cytosol. As is discussed in detail below, extensive unfolding of polypeptides is prerequisite for translocation. Preproteins in the cytosol are usually complexed with protein factors which protect them from aggregation and from acquiring a transportincompetent conformation [6, 11, 45]. Some of these factors, in particular heat-shock proteins of the hsp 70 class, require ATP for release. Chimeric proteins consisting of a mitochondrial preprotein and mouse cytosolic dihydrofolate reductase (DHFR) can be readily imported into mitochondria even when the DHFR domain is in a native folded state (because the mitochondrial import system can unfold the DHFR [50]). When, however, the DHFR domain is stabilized by adding methotrexate, a folate antagonist, import is blocked [13]. Under this condition, chimeric preproteins are translocated with their N-terminus, but translocation becomes arrested when the folded DHFR reaches the obstacle of the OM.

These observations led to the concept that the translocation machinery in the $\mathrm{OM}$ discussed above and the putative translocation machinery in the IM are in close contact during preprotein transfer. Previous observations had suggested that translocation can occur at sites of close proximity between OM and IM. Morphological contact sites were described several years ago [21]. Preproteins in transit were localized to these sites by electron microscopy after binding of antibodies and protein A-gold $[56,59,70]$. Thus, the question arose as to whether the two translocation systems form a stable complex, or whether the nature of their interaction is more dynamic.

The mitochondrial IMS contains a number of proteins whose precursors neither contain N-terminal signals nor need a $\Delta \Psi$ for import, but which do require the receptor/general insertion pore complex. Examples of such proteins are the cytochrome $c$ heme lyase and the cytochrome $c_{1}$ heme lyase, enzymes which catalyze the covalent attachment of the heme group to the respective apoproteins following their translocation into the IMS $[39,71]$. Thus, they can use the OM machinery in a selective fashion. This notion is underlined by the following observations. When a chimeric protein consisting of the presequence of $F_{1}$-ATPase subunit $\beta\left(F_{1} \beta\right)$ and cytochrome $c$ heme lyase was imported into mitochondria in the presence of a $\Delta \Psi$, it was localized to the matrix space; in contrast however, in the absence of a $\Delta \Psi$ it was localized to the IMS [60]. This is easily explained by the differential usage of the import signal in the presequence and in the cytochrome $c$ heme lyase moiety, respectively. Interestingly, when the chimeric protein was first accumulated in the IMS in the absence of a $\Delta \Psi$, and then in a second step a $\Delta \Psi$ was reestablished, import into the matrix space occurred. Apparently the transport systems in the two membranes can be used independently of each other in a consecutive manner $[19,51]$.

Highly purified OMs from $N$. crassa were prepared, and their ability to import preproteins was analyzed [43]. These outer membrane vesicles (OMV) were able to efficiently import OM proteins such as porin or the MOM components described above. They could also import cytochrome $c$ heme lyase with reasonable efficiency, but they were totally incompetent in the import of preproteins destined to the matrix. Most likely, a driving force exists in these vesicles for inserting proteins into the OM, which could be provided by the conformational alteration upon integration into the lipid phase of the membrane. However, such a driving force apparently does not exist in OMV for matrixtargeted preproteins. It should be emphasized that also in intact mitochondria import of matrixtargeted precursors into the IMS has never been observed in the absence of a $\Delta \Psi$, i.e., when translocation across the IM could not be initiated. (The energetics of translocation into the matrix are discussed in detail in the next section). 
It has also been found that mitoplasts, i.e., mitochondria in which the OM has been disrupted and partly removed, the import of various preproteins can take place [33]. This supports the view that also the IM translocation system on its own can translocate proteins. It is, however, not entirely clear whether the latter reaction is completely independent of the OM system. Recently, a few components of the IM have been defined mainly by genetic techniques which appear to be somehow involved in the transport process [10, 14, 31, 40]. The functional characterization of these components is presently a major effort in the field of mitochondrial targeting.

In summary, our present view of the interaction of the two translocation systems is that they are normally tightly coupled during the transport of preproteins destined to the matrix and the IM; however, this does not appear to be obligatory. It seems possible that they form "concerted channels" in a dynamic fashion when translocation is initiated at the surface. This coupling could be facilitated by the existence of morphological contact sites which bring the two membranes sufficiently close together. On the other hand, the OM system can be used in a selective manner to transfer proteins into the OM and the IMS.

\section{Energetics of translocation into the matrix: a central role for mitochondrial hsp70}

Two forms of energy are a prerequisite for the import of precursor proteins into mitochondria. ATP hydrolysis is required in the cytosol for release of protein factors bound to preproteins, and an electrical membrane potential $\Delta \Psi$ is necessary for translocation across the IM [16, 55] (see also Fig. 1). Whereas the first requirement can be circumvented by preproteins which do not have such factors bound, or when such factors are experimentally removed in vitro [5], the requirement for $\Delta \Psi$ is absolute, at least for preproteins targeted to the matrix and into the IM. However, $\Delta \Psi$ is not necessary for the translocation of the complete preprotein chain, but only for that of the $\mathrm{N}$-terminal presequence. Once the presequence is translocated, $\Delta \Psi$ can be dissipated without interfering with the completion of translocation [7, 55, 59].

What then is the driving force for translocating the main part of a preprotein across the two membranes? A few years ago we presented a hypothetical model which explains the mechanism of translocation and incorporates the energetic requirements observed for this process [46]. This

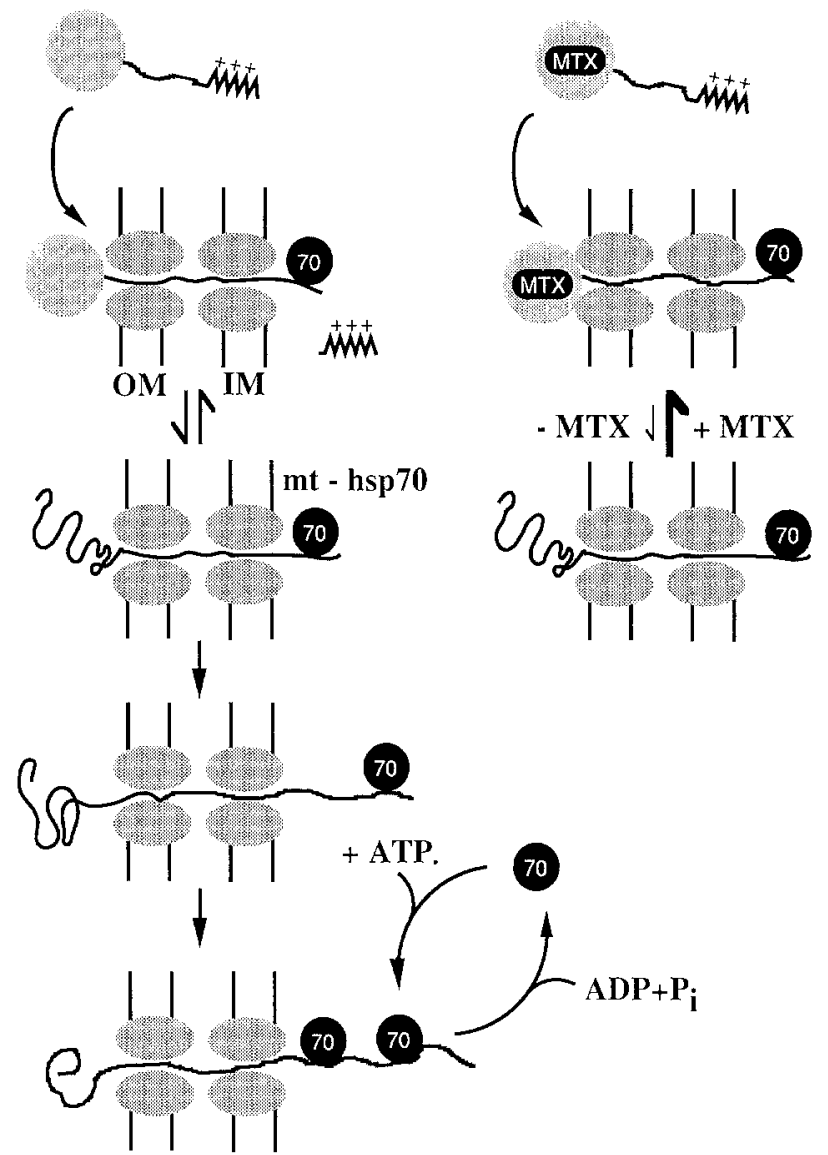

Fig. 4. Role of mitochondrial hsp70 in translocation of preproteins into the matrix. Import of a fusion protein between a mitochondrial preprotein and mouse cytosolic dihydrofolate reductase $(D H F R)$. Full circle, the folded DHFR in the preprotein. $M T X$, Methotrexate; 70, mt-hsp70

model is discussed below in the light of the most recent results.

There are three postulates in this model: (a) Preproteins can cross the mitochondrial membranes, in particular the IM, only in an unfolded, i.e., largely extended state. (b) Translocation is driven by repeated cycles of ATP-dependent binding of mt-hsp70 in the matrix to incoming unfolded segments of the preprotein (and ATP hydrolysis-dependent release of mt-hsp70). (c) Binding of mthsp70 facilitates the unfolding of preproteins on the mitochondrial surface since this shifts the equilibrium of folded and (partially) unfolded states which is due to spontaneous "breathing" of protein domains. A summary of this model is depicted in Fig. 4.

Observations showing that preproteins must become unfolded before or during import have been reported. It is difficult from these findings, however, to learn the degree to which the unfolding must occur. In particular one wishes to know the 
conformation of the polypeptide chain that is spanning the translocation pores in both membranes. There is no direct approach to answering this question; however, we have approached the problem by determining the minimal length of the spanning segment [52]. A series of chimeric preproteins were constructed containing N-terminal parts of different lengths of precytochrome $b_{2}$ fused to DHFR. These chimeric proteins could be all imported into isolated mitochondria and became arrested if methotrexate was present to prevent unfolding of the DHFR domain. The shortest chimeric protein that became translocated and processed by the MPP in the presence of methotrexate was one in which 55 amino acid residues were present between the MPP cleavage site and the DHFR domain. The distance across both mitochondrial membranes is about $20 \mathrm{~nm}$. This suggests that the spanning polypeptide was in a rather extended conformation. Although these data do not prove that preproteins must always be in an extended state to be able to cross the import pores, this seems a likely possibility.

\section{Conformation of preproteins during transit}

The question of which conformation is acquired by a polypeptide chain in transit is also of considerable relevance for the problem of how the IM can be penetrated by a macromolecule without its selective ion permeability being disturbed. A proton gradient exists across the IM. How does the IM remain sealed for protons when a polypeptide is crossing it? We have observed that no disturbance of the proton gradient occurs even when in a preprotein is accumulated in intact yeast cells in a membrane-spanning fashion to such a degree that the majority of import sites are occupied [70]. At the present time one can only speculate that the translocation pore in the IM is constructed in such a manner that it forms a "seal" around the chain in transit. How this can occur in view of the different nature of the amino acid side chains remains an absolute mystery.

\section{Mitochondrial hsp 70}

The essential role of $\mathrm{mt}-\mathrm{hsp} 70$ in import became apparent through the analysis of mutants in the gene for this mitochondrial member of the hsp 70 family which in yeast comprises at least eight proteins. The temperature-sensitive mutant initially investigated, ssc1-2, accumulated preproteins in vivo at the non-permissive temperature [34, 48]. Isolated mitochondria accumulated intermediates spanning the two membranes which were processed by MPP. This arrest could be overcome in the case of some precursors when these were unfolded prior to import. The mt-hsp 70 was found in association with preproteins in transit. More recently a more stringent temperature-sensitive mutant was analyzed, $s s c 1-3$, in which even the translocation of the presequence was blocked [15]. It appears that in the ssc1-2 mutant binding of mthsp70 was still possible although most probably with lower affinity. Interaction of $\mathrm{mt}-\mathrm{hsp} 70$ with polypeptide chains in transit was also demonstrated in wild-type yeast cells by chemical cross-linking and immunoprecipitation $[42,54]$.

\section{Role of matrix $A T P$}

The hsp70 function is dependent on ATP. It is most likely that the ATP form of mt-hsp70 is competent for preprotein binding, and that ATP hydrolysis is required for release of bound $\mathrm{mt}$-hsp 70 . Indeed a distinct requirement for ATP in the matrix has been described for the import of preproteins into the matrix $[32,67]$. Different levels of ATP depletion had different effects [67]. Drastic reduction to micromolar concentrations had virtually the same phenotype as the ssc1-3 mutation. Under these conditions even translocation of the presequence was inhibited, and precursors accumulated at the surface of the mitochondria [9]. When matrix ATP was reduced to intermediate levels accumulation of intermediates spanning both membranes was observed, also that of intermediates which were arrested in translocation across the IM but where the rest of the polypetide chain had crossed the OM. Apparently upon reduction of the driving force across the IM preproteins can slowly pass through the OM machinery. In summary, the observations on matrix ATP requirement are fully consistent with the role of ATP in regulating mt-hsp70 activity.

The requirement of mt-hsp70 for translocation of $\mathrm{N}$-terminal segments which was seen in the analysis of the matrix ATP requirement and of the ssc13 mutant suggests that the membrane potential alone is not sufficient to stably translocate the presequence. Rather, it appears that mt-hsp70 must bind either to the presequence or to parts immediately following the presequence. Therefore an intermediate in the import pathway was shown to accumulate in the absence of mt-hsp 70 function, but the presence of $\Delta \Psi$ which obviously had interacted with the IM translocation machinery but still needed a $\Delta \Psi$ when mt-hsp 70 function was reestablished [9]. 


\section{Unfolding of preproteins}

Results described so far support the concept that binding of mt-hsp70 to incoming preproteins favors unfolding on the outside by shifting the folding equilibrium. Direct evidence for such a role of mt-hsp70/ATP came from a series of observations on import of preproteins into the IMS which possess a dual targeting signal [67, 69]. Preproteins such as precytochrome $c_{1}$ and precytochrome $b_{2}$ have an $\mathrm{N}$-terminal matrix targeting signal followed by a sorting signal which directs the protein into the IMS. Two different pathways are presently being proposed to explain how these proteins enter the IMS, the so-called "conservative sorting mechanism" and the "stop-transfer mechanism" [20, 23]. Irrespective of how these precursors are sorted analysis of the energy and $\mathrm{mt}-\mathrm{hsp} 70$ requirements opened the possibility to study the role of mt-hsp70 in unfolding of domains during translocation. The $\mathrm{mt}$-hsp70/ATP was found not to be essential for the import of certain preproteins with dual targeting signals; apparently the import system directing the protein into the IMS provides the driving force. Precytochrome $b_{2}$, on the other hand does require mt-hsp70/ATP for import into the IMS. Cytochrome $b_{2}$ contains a cytochrome $b_{5}$-like heme binding domain which folds tightly in the preprotein in the cytosol, in particular in the presence of heme. In three different approaches it was shown that mt-hsp70/ATP is required to unfold this domain: matrix-ATP was dispensible for import when the protein was unfolded prior to import by $8 \mathrm{M}$ urea, or when the heme binding domain was partly or completely removed by recombinant DNA techniques, and precytochrome $b_{2}$ could be imported into a ssc1 mutant when heme was absent from the import assay $[34,67,69]$.

The experimental results described here are all in very good agreement with the postulates presented at the beginning of this contribution, and they strongly support the mechanism presented by the scheme in Fig. 4. It should be emphasized that this model is the first that is able to explain the mechanism and energetics of protein translocation across membranes on the basis of simple biochemical reactions. On the other hand, analysis of protein transport through the membranes of the endoplasmic reticulum and of the export of proteins from $E$. coli has identified quite a number of components directly and indirectly involved, and the availability of reconstituted systems may soon provide detailed insights into both the mechanism of translocation as well as into the energetics of the process.

\section{Folding of newly imported proteins in the matrix}

How do polypeptides fold after having reached the matrix space, and how do they assemble to macromolecular structures? Since the pioneering work of Anfinsen et al. [1] on the folding of ribonuclease, it had been a dogma that the information for the correct folding is contained in the amino acid sequence. This led to the general belief in recent decades that folding proteins do not even need help by other cellular components to achieve their native state. Studies on the folding of proteins imported into mitochondria challenged this view.

Mitochondria of the mutant mif4 of $S$. cerevisiae were found to import proteins almost as efficiently as wild-type mitochondria; however, the proteins did not achieve a functional state [8]. For instance, subunits of the enzyme ornithine transcarbamoylase did not form the trimeric enzyme, and the subunit $F_{1 \beta}$ was not incorporated into the $F_{1}$-ATPase complex. The gene affected in the mif4 mutant was that of hsp60, the mitochondrial homolog of E. coli GroEL. The prokaryotic GroEL protein was discovered as a component required for the assembly of phage $\lambda[28,30]$. A homologous protein, Rubisco binding protein, was detected in chloroplasts and was shown to associate with the large subunits of the enzyme ribulose bisphosphate carboxylase in the absence of the small subunits which together form the active enzyme [4]. The observations with hsp60 suggested that this protein, at least in eukaryotic cells, has a more general role in the assembly of proteins. Biochemical studies with mitochondrial hsp60 then revealed that this "chaperonin" is necessary for the folding of proteins [47]. The DHFR moiety of the chimeric preprotein pSu9DHFR protein (containing the presequence of subunit 9 of $\mathrm{F}_{1} \mathrm{~F}_{0}$-ATPase) was found to fold in association with hsp60 in an ATP-dependent manner. A large number of studies in vivo, in organello, and with purified reconstituted systems since then has established the general function of hsp60 in mitochondria and of the prokaryotic GroEL protein as chaperonins, i.e., as a factor which facilitates the correct and efficient folding of monomeric proteins which then allows the oligomerization to enzymatically active enzymes (for reviews see [18, 27]; Fig. 5).

These findings imply that perhaps not all but certainly a large number of imported proteins cannot simply be released from hsp70 as correctly folded monomers. Rather these proteins must be transferred to hsp60. How is this achieved? We have recently identified and characterized a component of the mitochondria, Mdj1p, which is required for 


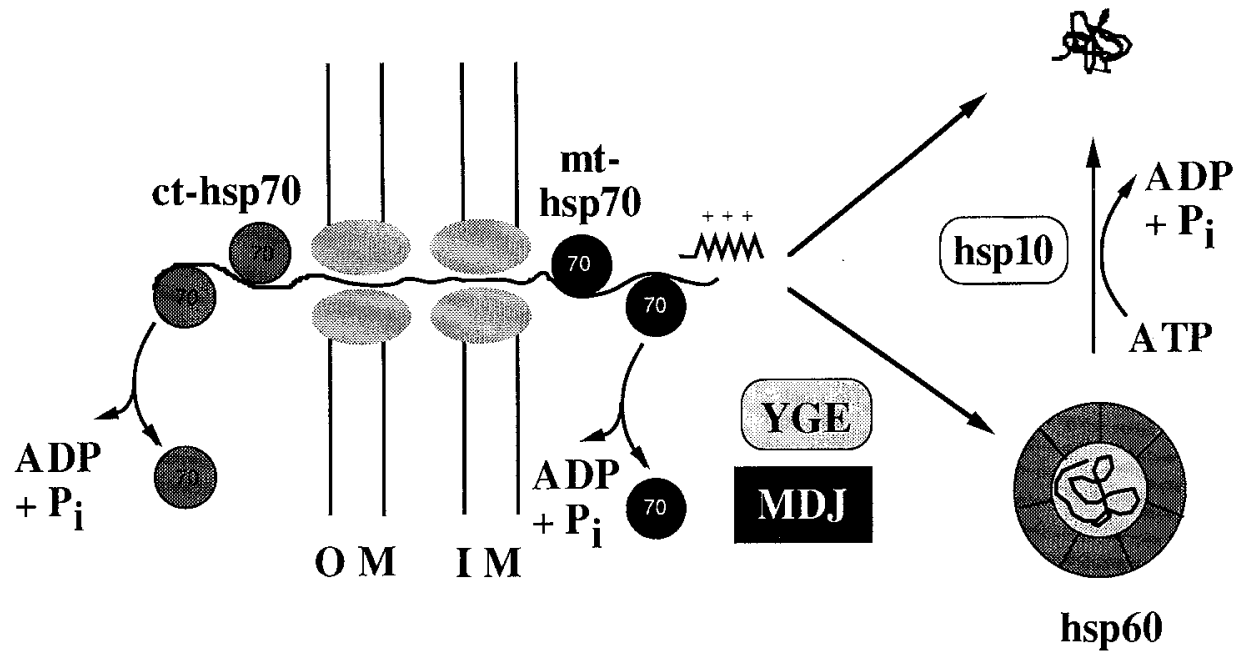

Fig. 5. Folding of proteins in mitochondria facilitated by hsp60. $M D J$, Mitochondrial DnaJ homolog; YGE, yeast GrpE equivalent; $h s p 10$, cochaperonin the folding of imported polypeptides as well as for the renaturation of unfolded proteins at elevated temperatures (Rowley et al., submitted for publication). Mdj1p is a heat shock protein as are hsp70 and hsp60. It is a member of the eukaryotic DnaJ family. DnaJ, on the other hand, is a bacterial heatshock protein which, together with DnaK (the bacterial hsp70 homolog) and GrpE have been found to be required for the replication of $\lambda$ phage DNA [17]. In the meantime, DnaK, DnaJ, and GrpE have been defined as a group of chaperones that cooperate in the handling of unfolded proteins or proteins with unfolded regions. They prevent the aggregation of unfolded proteins, facilitate refolding and prevent heat denaturation $[38,58,61]$. Reconstitution experiments have further suggested that after binding to unfolded proteins they can facilitate the transfer to GroEL [38]. The yeast deletion mutant, $\Delta$ mdj, which has a reduced ability in folding and refolding of imported preproteins, however, was not affected in its ability to import proteins (Rowley et al., submitted for publication). It therefore appears that Mdj1p does not cooperate with $\mathrm{mt}-\mathrm{hsp} 70$ in the translocation of preproteins, at least not in an obligatory manner, but is involved in passing on imported precursors from mthsp70 to hsp60. Interestingly, there is a preliminary report that yeast mitochondria also contain a GrpE homolog which is essential for the viability of the cells (E. Craig, personal communication).

The amazing similarities of the chaperone system of mitochondria and bacteria are another impressive illustration of how the prokaryotic traits of mitochondria have been conserved during evolution. Even more exciting, the mitochondrial system, in a novel type of reaction, appears to take advantage of the existing chaperone DnaK/hsp70 to cat- alyze the translocation of proteins. It is not known whether the prokaryotic ancestors of mitochondria originally contained a complex homologous to the mitochondrial protein import system. Such a putative complex following endosymbiosis would have evolved to deal with the import of those proteins now encoded by the host genome. Unfortunately, however, evolution does not always lend itself to direct experimental testing.

\section{Conclusions}

This review has discussed the fate of a mitochondrial protein destined to the matrix after its synthesis on and release from cytosolic ribosomes. There are a host of additional questions which have not been addressed. How are proteins sorted into the various subcompartments of the mitochondrion? How are proteins inserted into OM and IM? How are proteins assembled with cofactors such as heme and flavin groups? How are preproteins proteolytically processed? Answers are available to some aspects of these questions but a full picture is yet to come. A central problem in the whole field, however, remains almost entirely mysterious, this being the biochemical nature of the translocation pore. Why does it allow penetration only of unfolded polypeptide chains? What is the environment of a chain in transit? How are membrane functions and, in particular, the membrane potential maintained during the passage of protein molecules as large as $150 \mathrm{kDa}$ ? Understanding of the molecular nature of these pore functions will be one of the most challenging problems in the field.

Acknowledgements. I am very grateful to Dr. Rosemary Stuart and Dr. Thomas Langer for help in preparing this manuscript. 
This work was supported by the Sonderforschungsbereich 184 , the Deutsche Forschungsgemeinschaft, a grant from the Human Frontier Science Program, the EC Network on "Dynamics of Membrane Protein Insertion and Folding," and the Fonds der Chemischen Industrie.

\section{References}

1. Anfinsen CB, Haber E, Sela M, White FH (1961) The kinetics of formation of native ribonuclease during oxidation of the reduced polypeptide chain. Proc Natl Acad Sci USA 47:1309-1314

2. Attardi G, Schatz G (1988) The biogenesis of mitochondria. Annu Rev Cell Biol 4:289-333

3. Baker K, Schaniel A, Vestweber D, Schatz G (1990) ISP42, a protein of the yeast mitochondrial outer membrane, is essential for protein import and cell viability. Nature 348:605-609

4. Barraclough R, Ellis RJ (1980) Protein synthesis in chloroplasts. IX. Assembly of newly-synthesized large subunits into ribulose bisphosphate carboxylase in isolated intact pea chloroplasts. Biochem Biophys Acta 608:19-31

5. Becker K, Guiard B, Rassow J, Söllner T, Pfanner K (1992) Targeting of a chemically pure preprotein to mitochondria does not require the addition of a cytosolic signal recognition factor. J Biol Chem 267:5637-5643

6. Caplan A, Cyr DM, Douglas MG (1992) YDJ1p facilitates polypeptide translocation across different intracellular membranes by a conserved mechanism. Cell 71:1143-1155

7. Chen W-J, Douglas M (1987) The role of protein structure in the mitochondrial import pathway: unfolding of mitochondrially bound precursors is required for membrane translocation. J Biol Chem 262:15605-15609

8. Cheng M, Hartl F-U, Martin J, Pollock R, Kalousek F, Neupert W, Hallberg E, Hallberg R, Horwich A (1989) Mitochondrial heat-shock protein hsp60 is essential for assembly of proteins imported into yeast mitochondria. Nature 337:620-625

9. Cyr D, Stuart RA, Neupert W (1993) A matrix-ATP requirement for presequence translocation across the inner membrane of mitochondria. J Biol Chem (in press)

10. Dekker PJ, Keil P, Rassow J, Maarse AC, Pfanner N, Meijer M (1993) Identification of MIM23, a putative component of the protein import machinery of the mitochondrial inner membrane. FEBS Lett 330:66-70

11. Deshaies R, Koch B, Werner-Washburne M, Craig E, Schekman R (1988) A subfamily of stress proteins facilitates translocation of secretory and mitochondrial precursor polypeptides. Nature 332:800-805

12. Dietmeier K, Zara V, Palmisano A, Palmieri F, Voos W, Schloßmann J, Moczko M, Kispal G, Pfanner N (1993) Targeting and translocation of the phosphate carrier p32 to the inner membrane of yeast mitochondria. J Biol Chem 268 (in press)

13. Eilers M, Schatz G (1986) Binding of a specific ligand inhibits import of a purified precursor protein into mitochondria. Nature 322:228-232

14. Emtage JL, Jensen RE (1993) MAS6 encodes an essential inner membrane component of the yeast mitochondrial protein import pathway. J Cell Biol 122:1003-1012

15. Gambill B, Voos W, Kang PJ, Miao B, Langer T, Craig EA, Pfanner K (1993) A dual role for mitochondrial heat shock protein 70 in membrane translocation of preproteins. J Cell Biol 123 (in press)

16. Gasser S, Ohashi A, Daum G, Böhni P, Gibson J, Reid G, Yonetani T, Schatz G (1982) Imported mitochondrial proteins cytochromes $b_{2}$ and $c_{1}$ are processed in two steps. Proc Natl Acad Sci USA 79:267-271
17. Georgopoulos C, Ang D, Liberek K, Zylicz M (1990) Properties of the Escherichia coli heat shock proteins and their role in bacteriophage $\lambda$ growth. In: Morimoto Tissieres RI, Georgpopoulos C (eds) Stress proteins in biology and medicine. Cold Spring Harbor, New York, pp 191-222

18. Gething M-J, Sambrook J (1992) Protein folding in the cell. Nature 355:33-45

19. Glick B, Wachter C, Schatz G (1991) Protein import into mitochondria: two systems act in tandem? Trends Cell Biol $1: 99-103$

20. Glick B, Brandt A, Cunningham K, Müller S, Hallberg R, Schatz $G$ (1992) Cytochromes $c_{1}$ and $b_{2}$ are sorted to the intermembrane space of yeast mitochondria by a stop-transfer mechanism. Cell 68:809-822

21. Hackenbrock C (1968) Chemical and physical fixation of isolated mitochondria in low-energy and high-energy states. Proc Natl Acad Sci USA 61:589-605

22. Harkness TA, Nargang FE, van der Klei I, Neupert W, Lill R (1994) J Cell Biol (in press)

23. Hartl F-U, Neupert W (1990) Protein sorting to mitochondria: evolutionary conservations of folding and assembly. Science 247:930-938

24. Hartl F-U, Pfanner N, Nicholson D, Neupert W (1989) Mitochondrial protein import. Biochim Biophys Acta 998:1-45

25. Hase T, Riezmann H, Suda K, Schatz G (1983) Import of proteins into mitochondria: nucleotide sequence of the gene for a $70-\mathrm{kd}$ protein of the yeast mitochondrial outer membrane. EMBO J 2:2169-2172

26. Heijne $G$ von (1986) Mitochondrial targeting sequences may form amphiphilic helices. EMBO J 5:1335-1342

27. Hendrick J, Hartl F-U (1993) Molecular chaperone function of heat-shock proteins. Ann Rev Biochem 62:349-384

28. Hendrix R (1979) Purification and properties of GroE; a host protein involved in bacteriophage assembly. $\mathrm{J}$ Mol Biol $129: 375-392$

29. Hines V, Brandt A, Griffith $G$, Horstmann $H$, Brültsch $H$, Schatz G (1990) Protein import into yeast mitochondria is accelerated by the outer membrane protein MAS70. EMBO J 9:3191-3200

30. Hohn T, Hohn B, Engel A, Wortz M, Smith PR (1979) Isolation and characterization of the host protein GroE involved in bacteriophage $\lambda$ assembly. J Mol Biol 129:359--373

31. Horst M, Jenö P, Kronidou NG, Bollinger L, Oppliger W, Scherer P, Manning-Krieg U, Jascur T, Schatz G (1993) Protein import into yeast mitochondria: the inner membrane import site protein ISP45 is the MPI1 gene product. EMBO J 12:3035-3041

32. Hwang S, Schatz G (1989) Translocation of proteins across the mitochondrial inner membrane, but not into the outer membrane, requires nucleotide triphosphates in the matrix. Proc Natl Acad Sci USA 86:8432-8436

33. Hwang S, Jascur T, Vestweber D, Pon L, Schatz G (1989) Disrupted yeast mitochondria can import precursor proteins directly through their inner membrane. J Cell Biol 109:487493

34. Kang P-J, Ostermann J, Shilling J, Neupert W, Craig E, Pfanner N (1990) Requirement for hsp70 in the mitochondrial matrix for translocation and folding of precursor proteins. Nature 348:137-142

35. Kassenbrock CK, Cao W, Douglas MG (1993) Genetic and biochemical characterization of ISP6, a small mitochondrial outer membrane protein associated with the protein translocation complex. EMBO J 12:3023-3034

36. Kiebler M, Pfaller R, Söllner T, Griffith G, Horstmann H, Pfanner N, Neupert W (1990) Identification of a mitochondrial receptor complex required for recognition and membrane insertion or precursor proteins. Nature 348:610-616 
37. Kiebler M, Keil P, Schneider H, van der Klei I, Pfanner N, Neupert W (1993) The mitochondrial receptor complex: a central role of MOM22 in mediating transfer of preproteins from receptors to the general insertion pore. Cell 74:483-492

38. Langer T, Lu C, Echols H, Flanagan J, Hayer MK, Hartl FU (1992) Successive action of DnaK, DnaJ and GroEL along the pathway of chaperone-mediated protein folding. Nature 356:683-689

39. Lill R, Stuart R, Drygas M, Nargang F, Neupert W (1992) Import of cytochrome $c$ heme lyase into mitochondria: a novel pathway into the intermembrane space. EMBO J $11: 449-456$

40. Maarse A, Bloom J, Grivell L, Meijer M (1992) MPI1, an essential gene encoding a mitochondrial membrane protein, is possibly involved in protein import into yeast mitochondria. EMBO J 11:3619-3628

41. Mahlke K, Pfanner N, Horwich A, Hartl F-U, Neupert W (1990) Sorting pathways of mitochondrial inner membrane proteins. Eur J Biochem 192:551-555

42. Manning-Krieg U, Scherer P, Schatz G (1991) Sequential action of mitochondrial chaperones in protein import into the matrix. EMBO J 10:3273-3280

43. Mayer A, Lill R, Neupert W (1993) Translocation and insertion of precursor proteins into isolated outer membranes of mitochondria. J Cell Biol 121:2233-2243

44. Moczko M, Dietmeier K, Söllner T, Segui B, Steger H, Neupert W, Pfanner K (1992) Identification of the mitochondrial receptor complex in S. cerevisiae. FEBS Lett 310:265-268

45. Murakami H, Pain D, Blobel G (1988) $70 \mathrm{~K}$ heat-shock related protein is one of at least two distinct cytosolic factors stimulating protein import into mitochondria. J Cell Biol 107:2051-2057

46. Neupert W, Hartl F-U, Craig EA, Pfanner N (1990) How do polypeptides cross the mitochondrial membranes? Cell 63:447-450

47. Ostermann J, Horwich A, Neupert W, Hartl F-U (1989) Protein folding in mitochondria requires complex formation with hsp60 and ATP hydrolysis. Nature 341:125-130

48. Ostermann J, Voss W, Kang P, Craig E, Neupert W, Pfanner $\mathrm{N}$ (1990) Precursor proteins in transit through mitochondrial contact sites interact with hsp 70 in the matrix. FEBS Lett 277:281-284

49. Pfanner N, Hoeben P, Tropschug M, Neupert W (1987a) The carboxy-terminal two-thirds of the ADP/ATP carrier polypeptide contains sufficient information to direct translocation to mitochondria. J Biol Chem 262:14851-14854

50. Pfanner N, Rassow J, Guiard B, Söllner T, Hartl F-U, Neupert W (1990) Energy requirements for unfolding and membrane translocation of precursor proteins during import into mitochondria. J Biol Chem 265:16324-16329

51. Pfanner N, Rassow J, van der Klei I, Neupert W (1992) A dynamic model of the mitochondrial protein import machin. ery. Cell 68:999-1002

52. Rassow J, Hartl F-U, Guiard B, Pfanner N, Neupert W (1990) Polypeptides traverse the mitochondrial envelope in an extended state. FEBS Lett 275:190-194

53. Roise D, Schatz G (1988) Mitochondrial presequences. J Biol Chem 263:4509-4511

54. Scherer P, Krieg U, Hwang S, Vestweber D, Schatz G (1990) A precursor protein partly translocated into yeast mitochondria is bound to a $70 \mathrm{kd}$ mitochondrial stress protein. EMBO J 9:4315-4322

55. Schleyer M, Neupert W (1982) Requirement of a membrane potential for the posttranslational transfer of proteins into mitochondria. Eur J Biochem 125:109-116

56. Schleyer M, Neupert W (1985) Transport of proteins into mitochondria: translocation intermediates spanning contact sites between inner and outer membranes. Cell 43:330-350

57. Schneider H, Söllner T, Dietmeier K, Eckerskorn C, Lottspeich F, Trülzsch K, Neupert W, Pfanner N (1991) Targeting of the master receptor MOM19 to mitochondria. Science 254:1659-1662

58. Schröder H, Langer T, Hartl F-U, Bukau B (1993) DnaK, DnaJ and GrpE form a cellular chaperone machinery capable of repairing heat-induced protein damage. EMBO J $12: 4137-4144$

59. Schwaiger M, Herzog V, Neupert W (1987) Characterization of translocation contact sites involved in the import of mitochondrial proteins. J Cell Biol 105:235-246

60. Segui-Real B, Kispal G, Lill R, Neupert W (1993) Functional independence of the protein translocation machineries in mitochondrial outer and inner membranes: passage of preproteins through the intermembrane space. EMBO J 12:2211-2218

61. Skowyra D, Georgopoulos C, Zylicz M (1990) The E. coli dnaK gene product, the hsp 70 homolog, can reactivate heatinactivated RNA polymerase in an ATP hydrolysis-dependent manner. Cell 62:939-44

62. Smagula C, Douglas M (1988) ADP/ATP carrier of S. cerevisiae contains a mitochondrial import signal between amino acids 72 and 111. J Cell Biochem 36:323-328

63. Söllner T, Griffith G, Pfaller R, Pfanner N, Neupert W (1989) MOM19, an import receptor for mitochondrial precursor proteins. Cell $59: 1061-1070$

64. Söllner T, Pfaller R, Griffith G, Pfanner N, Neupert W (1990) A mitochondrial import receptor for the ADP/ATP carrier. Cell 62:107-115

65. Söllner T, Rassow J, Wiedmann M, Schlossmann J, Keil P, Neupert W, Pfanner N (1992) Mapping of the protein import machinery in the mitochondrial outer membrane by crosslinking of translocation intermediates. Nature 255:8487

66. Steger H, Söllner T, Kiebler M, Dietmeier K, Trülzsch K, Tropschug M, Neupert W, Pfanner N (1990) Import of ADP/ ATP carrier into mitochondria: two receptors act in parallel. J Cell Biol 111:2353-2363

67. Stuart R, Gruhler A, van der Klei I, Guiard B, Koll H, Neupert W (1994) The role of matrix ATP in the import of mitochondrial precursor proteins. Eur J Biochem (in press)

68. Vestweber D, Brunner K, Baker A, Schatz G (1989) A 42 K outer membrane protein is a component of the yeast mitochondrial import site. Nature 341:205-209

69. Voos W, Gambill BD, Guiard B, Pfanner K, Craig EA (1993) Presequence and Mature Part of Preproteins Strongly Influence the Dependence of Mitochondrial Protein Import on Heat Shock Protein 70 in the Matrix. J Cell Biol 123 (in press)

70. Wienhues U, Becker K, Schleyer M, Guiard B, Tropschug M, Horwich A, Pfanner N, Neupert W (1991) Protein folding causes an arrest of preprotein translocation in mitochondria in vivo. J Cell Biol 115:1601-1609

71. Zollner A, Rödel G, Haid A (1992) Molecular cloning and characterization of the Saccharomyces cerevisiae CYT2 gene encoding cytochrome- $c_{1}$ heme lyase. Eur J Biochem 207: $1093-1100$

72. Zwizinski C, Schleyer M, Neupert W (1983) Transfer of proteins into mitochondria: precursor of the ADP/ATP carrier binds to receptor sites on isolated mitochondria. J Biol Chem 258:4071-4074

73. Zwizinski C, Schleyer M, Neupert W (1984) Proteinaceous receptors for the import of mitochondrial precursor proteins. J Biol Chem 259:7850-7856 